\title{
COVID-19: Looking Into the Overlooked
}

\author{
Fernanda Cristina Petersen ${ }^{1 *}$, Ulf Reidar Dahle ${ }^{2}$, Belinda Nicolau ${ }^{3}$ and \\ Climent Casals-Pascual ${ }^{4,5}$ \\ ${ }^{1}$ University of Oslo, Oslo, Norway, ${ }^{2}$ Centre for Antimicrobial Resistance, Norwegian Institute of Public Health, Oslo, Norway, \\ ${ }^{3}$ Faculty of Dentistry, McGill University, Montreal, QC, Canada, ${ }^{4}$ Laboratorio de Microbiología, Centro de Diagnóstico \\ Biomédico, Hospital Clínic de Barcelona, Barcelona, Spain, ${ }^{5} \mathrm{Hospital}$ Clínic de Barcelona, Barcelona, Spain
}

Keywords: COVID- 19, microbiome, antibiotic resistance, SARS-CoV- 2, biomarker

\section{OPEN ACCESS}

Edited by:

Paola Patrignani,

University of Studies G. d'Annunzio

Chieti and Pescara, Italy

Reviewed by:

Stefania Tacconelli,

University of Studies G. d'Annunzio

Chieti and Pescara, Italy

Uma Gaur,

University of Macau, China

*Correspondence:

Fernanda Cristina Petersen

f.c.petersen@odont.uio.no

Specialty section:

This article was submitted to

Molecular Diagnostics and

Therapeutics,

a section of the journal

Frontiers in Molecular Biosciences

Received: 27 May 2020

Accepted: 29 June 2020

Published: 11 August 2020

Citation:

Petersen FC, Dahle UR, Nicolau B and Casals-Pascual C (2020) COVID-19:

Looking Into the Overlooked.

Front. Mol. Biosci. 7:165.

doi: 10.3389/fmolb.2020.00165
Viral infections have plagued humanity since the beginning of time causing numerous deaths through specific pathogenic events directly related to the virus and indirectly, through secondary infections accompanying or following the viral episode. Highly pathogenic bacteria, such as Neisseria meningitidis, Streptococcus pneumoniae, and Haemophilus influenzae can overgrow during viral infections, and cause severe bacterial infections. During the 1918 Spanish influenza pandemic, for instance, the majority of influenza fatalities were likely caused by secondary pneumococcal pneumonia (Morens et al., 2008), but we were not aware of it until 2008, when tissue biopsy investigations revealed this important association. Secondary bacterial infections also accounted for $25-50 \%$ of deaths during the 2009 H1N1 influenza pandemic (Macintyre et al., 2018). In this instance, the association with secondary infections, particularly with S. pneumoniae, was acknowledged only almost 10 years after the pandemic.

In the current COVID-19 pandemic, the role of co-infectiveness remains unclear. At the peak of the pandemic, broad-spectrum antibiotics have been administered to the majority of patients admitted with COVID-19 to hospital to prevent secondary infections but also, some antibiotics, like teicoplanin, have been used due to their alleged antiviral properties. In an early report, secondary infections were detected in 1 out of 7 patients. Among those that died, 50\% had secondary infections (Zhou F. et al., 2020). Other reports indicate that bacterial infections were documented in $<10 \%$ of COVID-19 patients (Zhou P. et al., 2020). In light of this uncertainty, antibiotic use has been reported to be as high as $74 \%$ among patients with COVID-19 who were admitted to ICUs (Cox et al., 2020). Some infections may have been caused by changes in colonization resistance, others due to increase use of corticosteroids, parenteral nutrition or just intravenous catheter infections. The administration of broad-spectrum antibiotics in the absence of high suspicion or documented infection challenges the current dogma of antimicrobial stewardship. This problem is further compounded by the fact that acute respiratory syndromes due to infection or non-infection are notoriously difficult to discriminate. However, the risk and potential severity of secondary infections associated with COVID-19, particularly in overcrowded clinical settings and in immune-compromised patients, has in many cases reduced the compliance with the local prescription practice guidelines.

A quick look at host factors may now be more relevant than ever. As the first wave of the pandemic is tailing off in Europe, we have gathered substantial and actionable evidence that suggests that both severity and mortality associated with COVID-19 is due to host factors, ranging from endothelial and coagulation disturbances leading to the formation of micro thrombi to a major dysregulation of the host immunity and inflammatory response. Here, a recent study has shown significant survival benefits of corticosteroids administration to patients with severe COVID-19 infection. 
How is the host contributing to the severity of COVID-19? It was a matter of time that a genome wide analysis study (GWAS) showed some association with disease severity. Indeed, we have recently learnt that particular blood groups (group A) may be linked to respiratory failure in COVID-19 patients (Ellinghaus et al., 2020). However, with a moderate increase severity risk (odds ratio: 1.45 with respect to other blood groups), it is unlikely that this association explain all the variability of disease severity. Here we should not forget that one of the major contributors that regulate individual immune/inflammatory responses is the human microbiome. In particular, the gut microbiome may influence directly and indirectly the immune response generated. Accordingly, the lymphocyte populations may adopt a more proinflammatory phenotype when microbiome diversity is reduced, as typically observed in old age patients (Figure 1) and recently reviewed by Dhar and Mohanty (2020).

Broad spectrum antibiotics are well-known to promote major disturbances in the microbiome. For critically ill patients, secondary infections and lack of response to antibiotics may be all that is necessary to shift the balance toward irreversible clinical deterioration and death. Also for some patients, antibiotics may do more harm than good. At least two of the antibiotics that are being used for treatment or prophylaxis in COVID19 patients, ceftriaxone and teicoplanin (Martinez, 2020), have been known since the 1990s to promote a substantial increase in the population of resistant enterococci in the gut microbiota (Meijer-Severs et al., 1990; Van Der Auwera et al., 1996). Azithromycin is another antibiotic that, like teicoplanin, has been repurposed for use in COVID-19 patients due to its possible antiSARS-CoV2 activity. Although there are few studies focusing on microbiota disturbances by azithromycin in adults, there are reports of macrolide resistance enrichment and reduction in diversity among patients with asthma (Taylor et al., 2019). Examples of promotion and suppression of viral infections in combination with antibiotics have been studied mostly in animal models. In humans, seminal studies have shown the impact on antibody response by approaches using antibiotic depletion of the microbiota in combination with live viral vaccines (Harris et al., 2018; Hagan et al., 2019).

The broad use of antibiotics during COVID-19 is per se an important reason to monitor changes in the microbiota of the respiratory or gastrointestinal tract. Moreover, in COVID-19, there are a number of striking observations in the course of the disease that we cannot yet explain, like healthy patients with minor or no co-morbidities that suddenly become irreversibly ill (Team, 2020). There are also more and more reports of patients cured with negative PCRs and positive antibodies that later on develop severe thrombotic events with reactivation of the virus (Oxley et al., 2020). Inflammatory responses are exacerbated in several cases, as indicated by cytokine

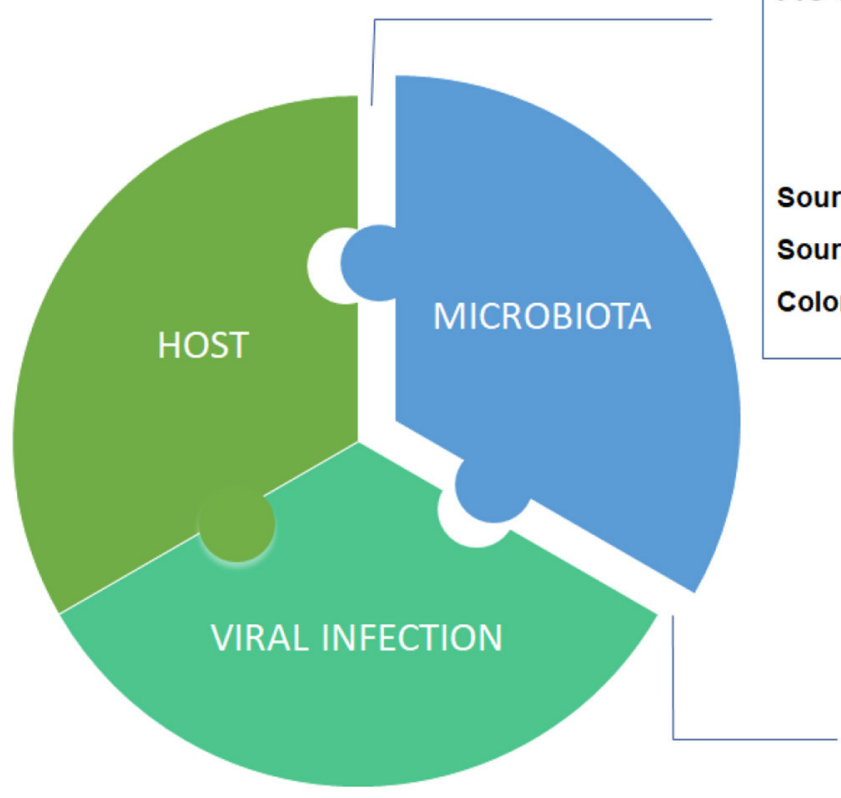

Potential to prevent or enhance viral dissemination via mechanisms such as:

Fucosin-like activity (capsid cleavage)

Enhancement of virion stability

Trapping of viruses

FIGURE 1 | Host-microbiota-virus triumvirate. Individual microbiome taxonomic profile and metabolic capacities may affect the balance between pro- and antiinflammatory responses, and between suppression or enhancement of viral dissemination. 
storms. Understanding the link between microbiome, immunity and inflammatory response in SARS-CoV-2 infected patients could help explaining such unexpected outcomes. A plethora of mechanisms by which the gut microbiota modulates the immune system have been described in the literature, and reviewed in several articles (Maynard et al., 2012; Honda and Littman, 2016; Levy et al., 2016). One relevant example are short chain fatty acids provided by the gut microbiota, which have a critical role in promoting expansion and differentiation of regulatory $\mathrm{T}$ cells (Tregs), thus impacting maintenance of immune homeostasis (Tanoue et al., 2016). In the airways, Tregs induced by short chain fatty acids contribute among other effects, to suppression of lung inflammation (Trompette et al., 2014). Both pro- and anti- inflammatory stimulation, and responses promoting or suppressing viral infections have been reported as directly modulated by members of the microbiota (Li et al., 2019). Of note, old age and comorbidities such as diabetes and cardiovascular diseases, which are linked to COVID-19 severity and mortality are also both associated with dysbiotic states (Peterson et al., 2015; Yang et al., 2015; Li et al., 2017).

Quantifying microbiota-host imbalances would have the potential to yield novel diagnostics for risk stratification and improved clinical management. However, implementing such clinical tools within a reasonable time frame depends on collecting this information from patients now. International consortia and multicenter studies have in record time organized initiatives to create and explore existing biobanks for COVID-19, as to understand the diversity in disease manifestations. Several risk indexes are created or combined with those commonly used in ICUs, and numerous initiatives to identify human genetic factors, antibody responses, and viral changes are underway, with virtually no mention on the microbiota. After two decades

\section{REFERENCES}

Cox, M. J., Loman, N., Bogaert, D., and O'grady, J. (2020). Co-infections: potentially lethal and unexplored in COVID-19. Lancet Microbe 1:e11. doi: 10.1016/S2666-5247(20)30009-4

Dhar, D., and Mohanty, A. (2020). Gut microbiota and Covid-19-possible link and implications. Virus Res. 2020:198018. doi: 10.1016/j.virusres.2020.198018

Ellinghaus, D., Degenhardt, F., Bujanda, L., Buti, M., Albillos, A., Invernizzi, P., et al. (2020). Genomewide Association Study of Severe Covid-19 with respiratory failure. N. Engl. J. Med. doi: 10.1056/NEJMoa2020283. [Epub ahead of print].

Hagan, T., Cortese, M., Rouphael, N., Boudreau, C., Linde, C., Maddur, M. S., et al. (2019). Antibiotics-driven gut microbiome perturbation alters immunity to vaccines in humans. Cell 178, 1313-1328 e1313. doi: 10.1016/j.cell.2019.08.010

Harris, V. C., Haak, B. W., Handley, S. A., Jiang, B., Velasquez, D. E., Hykes, B. L. Jr., et al. (2018). Effect of antibiotic-mediated microbiome modulation on rotavirus vaccine immunogenicity: a human, randomizedcontrol proof-of-concept trial. Cell Host Microbe 24, 197-207 e194. doi: 10.1016/j.chom.2018.07.005

Honda, K., and Littman, D. R. (2016). The microbiota in adaptive immune homeostasis and disease. Nature 535, 75-84. doi: 10.1038/nature18848

Levy, M., Thaiss, C. A., and Elinav, E. (2016). Metabolites: messengers between the microbiota and the immune system. Genes Dev. 30, 1589-1597. doi: $10.1101 / \operatorname{gad} .284091 .116$

Li, N., Ma, W. T., Pang, M., Fan, Q. L., and Hua, J. L. (2019). The commensal microbiota and viral infection: a comprehensive of major milestone discoveries in the field, this is somehow surprising. So far, search for COVID-19 biobank initiatives at Biobanks Europe (https://www.bbmri-eric.eu) do not retrieve any hits for fecal samples. Actually, as of May 27th 2020, only seven in 1706 registries of COVID-19 studies in the ClinicalTrials.gov refers to microbiome or microbiota as an outcome measurement.

"The art of being wise is the art of knowing what to overlook"- William James, 1890

Focusing either on the host or on the virus during pandemics may be the wise way to go when resources are scarce and priorities must be established. On the other hand, the history of human infections, and the accumulated knowledge on the interplay of the human microbiota and the immune system indicate that we may be overlooking one of the three core elements in the present COVID-19 pandemic. With COVID-19 varying in severity from asymptomatic to lethal, the microbiota could provide valuable biomarkers to predict which individuals are most at risk of suffering severe disease.

\section{AUTHOR CONTRIBUTIONS}

FP wrote the paper with inputs from all authors. All authors conceived the presented ideas and approved the final manuscript.

\section{FUNDING}

This work was supported by a grant from the Norwegian Research Council (Grant numbers-273833 and 274867), and the Olav Thon Foundation. BN holds a Canada Research Chair in Life Course Oral Epidemiology. review. Front. Immunol. 10:1551. doi: 10.3389/fimmu.2019. 01551

Li, X., Watanabe, K., and Kimura, I. (2017). Gut microbiota dysbiosis drives and implies novel therapeutic strategies for diabetes mellitus and related metabolic diseases. Front. Immunol. 8:1882. doi: 10.3389/fimmu.2017. 01882

Macintyre, C. R., Chughtai, A. A., Barnes, M., Ridda, I., Seale, H., Toms, R., et al. (2018). The role of pneumonia and secondary bacterial infection in fatal and serious outcomes of pandemic influenza a(H1N1)pdm09. BMC Infect. Dis. 18:637. doi: 10.1186/s12879-018-3548-0

Martinez, M. A. (2020). Compounds with therapeutic potential against novel respiratory 2019 Coronavirus. Antimicrob Agents Chemother 64: e00399-20. doi: 10.1128/AAC.00399-20

Maynard, C. L., Elson, C. O., Hatton, R. D., and Weaver, C. T. (2012). Reciprocal interactions of the intestinal microbiota and immune system. Nature 489, 231-241. doi: 10.1038/nature11551

Meijer-Severs, G. J., Van Santen, E., and Meijer, B. C. (1990). Shortchain fatty acid and organic acid concentrations in feces of healthy human volunteers and their correlations with anaerobe cultural counts during systemic ceftriaxone administration. Scand. J. Gastroenterol. 25, 698-704. doi: 10.3109/003655290089 97595

Morens, D. M., Taubenberger, J. K., and Fauci, A. S. (2008). Predominant role of bacterial pneumonia as a cause of death in pandemic influenza: implications for pandemic influenza preparedness. J. Infect. Dis. 198, 962-970. doi: $10.1086 / 591708$ 
Oxley, T. J., Mocco, J., Majidi, S., Kellner, C. P., Shoirah, H., Singh, I. P., et al. (2020). Large-vessel stroke as a presenting feature of covid19 in the young. N. Engl. J. Med. 382:e60. doi: 10.1056/NEJMc20 09787

Peterson, C. T., Sharma, V., Elmén, L., and Peterson, S. N. (2015). Immune homeostasis, dysbiosis and therapeutic modulation of the gut microbiota. Clin. Exp. Immunol. 179, 363-377. doi: 10.1111/cei.12474

Tanoue, T., Atarashi, K., and Honda, K. (2016). Development and maintenance of intestinal regulatory $\mathrm{T}$ cells. Nat. Rev. Immunol. 16, 295-309. doi: $10.1038 /$ nri.2016.36

Taylor, S. L., Leong, L. E. X., Mobegi, F. M., Choo, J. M., Wesselingh, S., Yang, I. A., et al. (2019). Long-term azithromycin reduces Haemophilus influenzae and increases antibiotic resistance in severe asthma. Am. J. Respir. Crit. Care Med. 200, 309-317. doi: $10.1164 / \mathrm{rccm} .201809-1739$ OC

Team, C. C.-R. (2020). Severe Outcomes Among Patients with Coronavirus Disease 2019 (COVID-19) - United States, February 12-March 16, 2020. MMWR Morb. Mortal Wkly. Rep. 69, 343-346. doi: 10.15585/mmwr.mm6912e2

Trompette, A., Gollwitzer, E. S., Yadava, K., Sichelstiel, A. K., Sprenger, N., Ngom-Bru, C., et al. (2014). Gut microbiota metabolism of dietary fiber influences allergic airway disease and hematopoiesis. Nat. Med. 20, 159-166. doi: $10.1038 / \mathrm{nm} .3444$

Van Der Auwera, P., Pensart, N., Korten, V., Murray, B. E., and Leclercq, R. (1996). Influence of oral glycopeptides on the fecal flora of human volunteers: selection of highly glycopeptide-resistant enterococci. J. Infect. Dis. 173, 1129-1136. doi: 10.1093/infdis/173.5.1129

Yang, T., Santisteban, M. M., Rodriguez, V., Li, E., Ahmari, N., Carvajal, J. M., et al. (2015). Gut dysbiosis is linked to hypertension. Hypertension 65, 1331-1340. doi: 10.1161/HYPERTENSIONAHA.115.05315

Zhou, F., Yu, T., Du, R., Fan, G., Liu, Y., Liu, Z., et al. (2020). Clinical course and risk factors for mortality of adult inpatients with COVID-19 in Wuhan, China: a retrospective cohort study. Lancet 395, 1054-1062. doi: 10.1016/S0140-6736(20)30566-3

Zhou, P., Liu, Z., Chen, Y., Xiao, Y., Huang, X., and Fan, X. G. (2020). Bacterial and fungal infections in COVID-19 patients: a matter of concern. Infect. Control Hosp. Epidemiol. 1-2. doi: 10.1017/ice.2020.156

Conflict of Interest: The authors declare that the research was conducted in the absence of any commercial or financial relationships that could be construed as a potential conflict of interest.

Copyright (C) 2020 Petersen, Dahle, Nicolau and Casals-Pascual. This is an openaccess article distributed under the terms of the Creative Commons Attribution License (CC BY). The use, distribution or reproduction in other forums is permitted, provided the original author(s) and the copyright owner(s) are credited and that the original publication in this journal is cited, in accordance with accepted academic practice. No use, distribution or reproduction is permitted which does not comply with these terms. 